\title{
Impact of Facility Delivery Intensity on the Practice of Active Management of Third Stage of Labour and Other Labour and Delivery Interventions in Tanzania
}

\author{
Hilda Kanama, Projestine Muganyizi* \\ Department of Obstetrics \& Gynecology, Muhimbili University of Health Sciences (MUHAS), Dar es Salaam, Tanzania \\ Email: hkanama@gmail.com, *promuga@yahoo.com
}

How to cite this paper: Kanama, H. and Muganyizi, P. (2019) Impact of Facility Delivery Intensity on the Practice of Active Management of Third Stage of Labour and Other Labour and Delivery Interventions in Tanzania. Open Journal of Obstetrics and Gynecology, 9, 730-744.

https://doi.org/10.4236/ojog.2019.95073

Received: February 18, 2019

Accepted: May 24, 2019

Published: May 27, 2019

Copyright $\odot 2019$ by author(s) and Scientific Research Publishing Inc. This work is licensed under the Creative Commons Attribution International License (CC BY 4.0).

http://creativecommons.org/licenses/by/4.0/

\begin{abstract}
Background: Postpartum haemorrhage ( $\mathrm{PPH})$ is the leading cause of maternal mortality globally and Tanzania particularly. The World Health Organisation recommends Active Management of Third Stage of Labour (AMTSL) in preventing $\mathrm{PPH}$, but its correct implementation has generally remained low. Many factors have been associated with the low practice of AMTSL, but the literature on the impact of delivery intensity is scanty. The aim was to determine the impact of facility delivery intensity on correct practice of AMTSL and provision of other labour and delivery interventions. Methods: In 2016, an analytical cross-section study was conducted in four public health facilities in Dar es Salaam. A priori criterion was set at 10 daily deliveries or more for High Delivery Intensity Facilities (HDIFs) in contrast to Low Delivery Intensity Facilities (LDIFs). All public obstetric care hospitals and health centres were ranked such that one facility with the highest deliveries and one with the lowest deliveries per category were selected. All eligible deliveries between $8.00 \mathrm{am}$ and $2.00 \mathrm{pm}$ were studied. An observer was assigned to each facility to time and document delivery and third stage events. Data were entered and analysed using SPSS program version 20. Pearson Chi square test was used for categorical data and Student's t-test for comparing continuous data. Clinical relevance of the differences was evaluated by using $95 \%$ confidence intervals. Statistics with p-value $<0.05$ were taken as significant. The study was ethically approved by MUHAS Senate Research and Publication Committee. Results: In total, 752 normal deliveries were observed including 376 in LDIFs and 376 in HDIFs. Socio-demographic characteristics of women were comparable in both, except that women with secondary or higher education were more delivered in LDIFs (60.4\%) than in HDIFs (35.1\%), p <
\end{abstract}


0.001. LDIFs were more likely to provide Oxytocin as recommended $(\mathrm{OR}=$ 4.0, 95\% CI: $3.01-5.25)$ and to perform other life saving interventions than the HDIFs. Conclusions: Facility delivery intensity has remarkable impact on correct practice of AMTSL, labor and intra-partum care. Efforts to reduce maternal deaths should minimize facility delivery intensity.

\section{Keywords}

Active Management, Third Stage, Delivery Intensity, Hemorrhage, Tanzania

\section{Introduction}

Obstetric hemorrhage remains the leading cause of maternal mortality in developed and developing countries. Severe hemorrhage after childbirth is the most common form of obstetric hemorrhage, being responsible for approximately a third of all maternal deaths worldwide [1]. In Tanzania, this cause is responsible for up to $39 \%$ of all maternal deaths [2] [3].

Most postpartum hemorrhage complications occur within 24 hours of childbirth and are typically due to atonic uterus in $70 \%$ of cases [4]. This type of obstetric hemorrhage can be effectively prevented by implementing Active Management of Third Stage of Labour (AMTSL) on all women who have given birth since such events are largely unpredictable [5]. According to the International Federation of Gynecology and Obstetrics (FIGO) and the International Confederation of Midwives (ICM), AMTSL entails the provision of the uterotonic agent after birth, controlled cord traction and uterine massage after delivery of the placenta [4]. However, a multi-centered Clinical trial studying the relevance of the individual AMTSL components concluded that administration of Oxytocic within 1 minute of childbirth is the single most important component of AMTSL [5] [6] [7]. Following these results and other existing evidence, WHO recommends AMTSL to all delivering women and a uterotonic agent administered within 1 minute of childbirth as the most important and indispensable component, while the other two components of AMTSL are optional [8]. In Tanzania, the National guidelines for use of uterotonics in AMTSL also recommend the use of Oxytocin within a minute of childbirth and mentions Ergometrine or Misoprostol as alternatives if Oxytocin is not available [9].

Despite the effectiveness of AMTSL in preventing postpartum hemorrhage, and the wide advocacy, adherence to AMTSL guidelines has been reported to vary widely. In a survey of 15 university teaching hospitals in 10 developed and developing countries, the practice of AMTSL was $25 \%$, being observed in $0 \%$ to $98 \%$ of deliveries regardless of the development status of the countries [10]. Among the observed deliveries in seven developing countries including Tanzania in 2005, the uterotonic agent was administered within one minute of birth to only $33.8 \%$ of the observed deliveries (ranging from $10.4 \%$ to $67.3 \%$ ) [11]. Overall in Tanzania, studies using nationally representative and regional samples in- 
dicate the correct uterotonic administration for AMTSL in 7\% to 50\% of the observed deliveries [11] [12] [13].

According to the World Health Report in 2006, Tanzania was one among the 57 countries in crisis for human resource for health, with fewer than 23 workforce (doctors, nurses, midwives) per 10,000 population [14]. The Primary Health Services Development Program (PHSDP) which was introduced in 2007 with the aim to establish and staff an additional 2074 health centres and 8 district hospitals by 2017 would lead to having a Health Centre at every ward and a District Hospital at every District [15]. While this program would decongest obstetric care facilities, the problem persists especially in urban areas and the impact of such high delivery intensity on implementing life saving procedures such as AMTSL remains unclear. This study compares adherence to the National guidelines on implementing Active Management of Third Stage of Labor (AMTSL) and other potentially lifesaving labor and delivery interventions in Low Delivery intensity facilities (LDIFs) in contrast to high delivery intensity facilities (HDIFs) in an urban setting. This study intends to inform policy makers, program managers and researchers on the implications of the current delivery intensities on proper implementation of some key life saving obstetric procedures at the time when the Primary Health Services Development Program is winding-up while maternal mortality remains persistently high.

\section{Methods}

This analytical cross-sectional study was conducted in February 2016 in Dar es Salaam Tanzania. Four public obstetric care facilities were selected based on their delivery intensities. From an official exhaustive list of 54 hospitals and health centers that provided obstetric care services in Dar es Salaam, 39 private facilities and the Muhimbili National Hospital were excluded. The remaining 15 facilities were visited and delivery records for the specified past 3 days were reviewed to establish the average daily number of deliveries. Hospitals and health centers were then ranked separately in the order of daily delivery intensities. From each of the two groups, one facility ranking the highest and one ranking the lowest were selected for the study. It was agreed a priori to take 10 deliveries per day as the cut-off point for HDIFs. Thus, two sets of facilities each with one health center and one hospital were enrolled according to level of delivery intensity.

Women who delivered in the study facilities were eligible for enrolment if they gave birth between 8.00 am and $2.00 \mathrm{pm}$, if the pregnancy was 28 weeks of gestation or above and if they delivered vaginally without instrumentation. In order to overcome low quality documentation of information, a parallel system of recording was introduced to each study facility involving timing and documenting intrapartum and third stage events to supplement the routine data for all laboring women. Healthcare providers were unaware of the actual primary study interest. The study was generally introduced to service providers as timing of the duration of second and third stage of labor. The enrolment of women into 
the study was made after delivery. All eligible women were asked for consent to use their routine records in the study and those who consented were enrolled. A sample size of 752 deliveries was calculated to allow comparison of administration of Oxytocin within 1 minute of birth in LDIFs and HDIFs. Based on the wide prevalence range of appropriate use of uterotonic for AMTSL in Tanzania of $7 \%$ to $50 \%$ [11] [12] [13], a reasonable assumption of $30 \%$ prevalence of correct uterotonic administration in LDIFs and $10 \%$ in the HDIFs was made. A sample size of 225 on each group for a two sided significance level with $\alpha$ probability set at $5 \%$ would give Power (1- $\beta$ ) of at least $90 \%$ to detect the difference. Adjusting for $10 \%$ non-response and a 1.5 design effect the sample size of 752 (376 on each group) was considered adequate.

Information about socio-demographic characteristics, past obstetric history, antenatal medical and obstetric complications for current pregnancy, initiation of current labor and monitoring events, medicines and fluids given during labor, timed duration of delivery events and practice of AMTSL was entered in the checklist. These data were obtained from case note records or directly from observation or interview with the women after delivery. Data were recorded on a checklist by data collectors who were intern doctors trained on how to perform AMTSL and on using the checklist (see appendix). These data collectors also participated in pre-testing of the checklist during which the correct observation approach, timing skills using a stop watch, obtaining information from facility records and correct filling of the checklist were fine tuned. Each facility was assigned a data collector who worked under supervision of the principle investigator. Three components of AMTSL were observed and recorded including the duration from time of delivery of the baby to injection of Oxytocin, application of controlled cord traction and performance of uterine massage [1] [4] [5] [7]. Data for all enrolled women were scrutinized up to 3 hours after delivery. Data analysis was done using SPSS version 20 statistical computer program. In comparing variables between LDIFs and HDIFs, Pearson Chi square test was used for categorical data and Student's t-test for continuous data. The clinical relevance of the differences was evaluated by the $95 \%$ Confidence Intervals. Statistics with $\mathrm{p}$-value $<0.05$ were taken as significant.

\section{Results}

In total, 752 deliveries from the four study facilities were observed, 376 from LDIFs and another 376 from HDIFs. Most women (62.9\%) were aged $20-29$ years with ages ranging from 15 to 45 years, and a median of 25.0 years. Overall, socio demographic characteristics of women who delivered in LDIFs and HDIFs did not differ significantly in terms of age distribution, marital and gravidity statuses on admission. However, significantly more women who are educated to secondary or higher levels delivered in LDIFs (60.4\%) than in HDIFs (35.1\%), $\mathrm{p}$ $<0.001$ (Table 1).

Interventions during labor and delivery are important in improving maternal and fetal outcomes. The practice of six of such interventions was studied and the 
results are presented in Table 2. Overall, significantly more interventions were practiced in LDIFs than HDIFs.

Comparing with deliveries in HDIFs, women who delivered in LDIFs were more than ten times likely their labor to be induced $(\mathrm{OR}=10.5, \mathrm{p}<0.001)$, more than 2 times likely to be catheterized $(\mathrm{OR}=2.5, \mathrm{p}<0.001)$ and more than three times likely to undergone episiotomy $(\mathrm{OR}=3.4, \mathrm{p}<0.001)$. Partograph filling was universally done in LDIFs as in HDIFs and the provision of intravenous fluids was comparable for the two groups (Table 2).

AMTSL was practiced in all the study facilities. In all the study facilities, Oxytocin was the only drug used for AMTSL and was ultimately given to all except two women. Overall, correct provision of Oxytocin within one minute of childbirth was practiced on 497 (66.3\%) of the observed deliveries. Other practiced, but less important components of AMTSL are the controlled cord traction and uterine massage (Table 3).

Among the 376 women who delivered in LDIFs, 85.6\% received Oxytocin within one minute of childbirth compared to only $46.5 \%$ of the 376 women who delivered in HDIF, thus giving a four times higher likelihood of receiving Oxytocin correctly in LDIFs than in HDIFs, $\mathrm{p}<0.001$. Likewise, it was overall more likely to have controlled cord traction and uterine massage done in LDIFs than HDIFs (Table 3).

Table 1. Socio-demographic Characteristics of the Delivered Women, $\mathrm{N}=752$.

\begin{tabular}{|c|c|c|c|c|c|c|}
\hline \multicolumn{7}{|c|}{ Facility Delivery Intensity } \\
\hline Characteristics & $\begin{array}{c}\text { All } \\
\text { n (\%) }\end{array}$ & $\begin{array}{l}\text { Low } \\
\text { n (\%) }\end{array}$ & $\begin{array}{l}\text { High } \\
\text { n (\%) }\end{array}$ & OR & $95 \% \mathrm{CI}$ & $\mathrm{p}$ value \\
\hline \multicolumn{7}{|l|}{ Age } \\
\hline $15-19$ & $96(12.8)$ & $41(10.9)$ & $55(14.6)$ & 1 & & 0.808 \\
\hline $20-29$ & $473(62.9)$ & $240(63.8)$ & $233(62.0)$ & 1.20 & $0.64-2.23$ & \\
\hline $30-45$ & $183(24.3)$ & $95(25.3)$ & $88(23.4)$ & 1.01 & $0.69-1.50$ & \\
\hline \multicolumn{7}{|l|}{ Marital status } \\
\hline Single & $124(16.5)$ & $68(18.1)$ & $56(14.9)$ & 1 & & 0.12 \\
\hline Married/cohabiting & $617(82)$ & $301(80.1)$ & $316(84.0)$ & 0.89 & $0.23-3.47$ & \\
\hline Divorced & $11(1.5)$ & $7(1.9)$ & $4(1.1)$ & 1.72 & $0.47-6.29$ & \\
\hline \multicolumn{7}{|l|}{ Level of education } \\
\hline Illiterate & $19(2.5)$ & $11(2.9)$ & $8(2.1)$ & 1 & & $<0.001$ \\
\hline Primary education & $374(49.7)$ & $138(36.7)$ & $236(62.8)$ & 1.40 & $0.54-3.65$ & \\
\hline $\begin{array}{c}\text { Secondary/Higher } \\
\text { education }\end{array}$ & $359(47.7)$ & $227(60.4)$ & $132(35.1)$ & 3.05 & $2.23-4.16$ & \\
\hline \multicolumn{7}{|l|}{ Gravidity } \\
\hline Primigravid & $286(38)$ & $130(34.6)$ & $156(41.5)$ & 1 & & 0.103 \\
\hline Gravida $2-4$ & $406(54)$ & $218(58.0)$ & $188(50.0)$ & 1.2 & $0.63-2.31$ & \\
\hline$\geq 5$ & $60(8)$ & $28(7.4)$ & $32(8.5)$ & 0.83 & $0.45-1.49$ & \\
\hline
\end{tabular}


Table 2. Comparison of labor and delivery interventions in low and high delivery Intensity Facilities.

\begin{tabular}{|c|c|c|c|c|c|c|}
\hline \multicolumn{7}{|c|}{ Facility Delivery Intensity } \\
\hline Characteristics & All & $\begin{array}{c}\text { Low } \\
\text { n (\%) }\end{array}$ & $\begin{array}{l}\text { High } \\
\text { n (\%) }\end{array}$ & OR & $95 \% \mathrm{CI}$ & $p$ value \\
\hline \multicolumn{7}{|l|}{ Initiation of labor } \\
\hline Spontaneous & $721(95.9)$ & $348(92.6)$ & $373(99.2)$ & 1 & & \\
\hline Induced & $31(4.1)$ & $28(7.4)$ & $3(0.8)$ & 10.5 & $3.1-35.8$ & $<0.001$ \\
\hline \multicolumn{7}{|l|}{ Labor augmented } \\
\hline Yes & $113(15)$ & $41(10.9)$ & $72(19.1)$ & 0.1 & $0.19-0.59$ & $<0.001$ \\
\hline No & $639(85)$ & $335(89.1)$ & $304(80.9)$ & 1 & & \\
\hline \multicolumn{7}{|l|}{ Partograph filled } \\
\hline Yes & $697(92.7)$ & $353(93.9)$ & $344(91.5)$ & 1.2 & $0.66-2.11$ & 0.565 \\
\hline No & $55(7.3)$ & $23(6.1)$ & $32(8.5)$ & 1 & & \\
\hline \multicolumn{7}{|c|}{ Urinary catheterization } \\
\hline Yes & $227(30.2)$ & $134(35.6)$ & $93(24.7)$ & 2.5 & $1.59-3.9$ & $<0.001$ \\
\hline No & $525(69.8)$ & $242(64.4)$ & $283(75.3)$ & 1 & & \\
\hline \multicolumn{7}{|l|}{ Episiotomy done } \\
\hline Yes & $83(11)$ & $61(16.2)$ & $22(5.9)$ & 3.4 & $2.0-5.8$ & $<0.001$ \\
\hline No & $669(89)$ & $315(83.9)$ & $354(94.1)$ & 1 & & \\
\hline \multicolumn{7}{|l|}{ Iv fluid given } \\
\hline Yes & $216(28.7)$ & $105(27.9)$ & $111(29.5)$ & 0.74 & $0.44-1.26$ & 0.268 \\
\hline No & $536(71.3)$ & $271(72.1)$ & $265(70.5)$ & 1 & & \\
\hline $\begin{array}{l}\text { Placenta checked } \\
\text { for completeness }\end{array}$ & & & & 1.62 & $0.40-6.54$ & 0.5 \\
\hline Yes & $743(98.8)$ & $372(98.9)$ & $371(98.7)$ & 1 & & \\
\hline No & $9(1.2)$ & $4(1.1)$ & $5(1.3)$ & 1.25 & $0.33-4.70$ & 0.74 \\
\hline
\end{tabular}

Table 3. The association of practice of AMTSL components with delivery in Low and High Delivery Intensity facilities in Dar es Salaam.

\begin{tabular}{|c|c|c|c|c|c|c|}
\hline \multirow[b]{2}{*}{ Characteristics } & \multirow[b]{2}{*}{ All } & \multicolumn{2}{|c|}{ Facility Delivery Intensity } & \multirow[t]{2}{*}{ OR } & \multirow[t]{2}{*}{$95 \% \mathrm{CI}$} & \multirow[t]{2}{*}{$\mathrm{p}$ value } \\
\hline & & Low & High & & & \\
\hline \multicolumn{7}{|l|}{$\begin{array}{l}\text { Oxytocin given } \\
\text { within } 1 \text { Minute }\end{array}$} \\
\hline Yes & $497(66.1)$ & $322(85.6)$ & $175(46.5)$ & 3.98 & $3.01-5.25$ & $<0.001$ \\
\hline No & $255(33.9)$ & $54(14.4)$ & $201(53.5)$ & 1 & & \\
\hline \multicolumn{7}{|l|}{$\begin{array}{l}\text { Controlled } \\
\text { cord traction }\end{array}$} \\
\hline Yes & $737(98.0)$ & $373(99.2)$ & $364(96.8)$ & 4.1 & $1.1-14.7$ & 0.019 \\
\hline No & $15(2.0)$ & $3(0.8)$ & $12(3.2)$ & & & \\
\hline \multicolumn{7}{|c|}{ Uterine massage done } \\
\hline Yes & $725(96.4)$ & $368(97.9)$ & $357(94.9)$ & 2.4 & $1.1-5.7$ & 0.031 \\
\hline No & $27(3.6)$ & $8(2.1)$ & $19(5.1)$ & & & \\
\hline
\end{tabular}




\section{Discussion}

Obstetric hemorrhage is the leading cause of maternal mortality globally and in Tanzania where up to $39 \%$ of maternal deaths result from this cause [3]. This study was conducted in the context of high maternal mortality, whose ratio is estimated at 556 deaths per 100,000 live births, and at the peak of government's efforts through its Primary Health Services Development Program (PHSDP) to construct and staff a large number of health centres and district hospitals by 2017 [15]. A successful PHSDP would have improved obstetric care facility density in the country and relieved congestion in obstetric care wards. The current study was therefore conducted at the time when congestion in obstetric care wards would be considered at its lowest level and maternal deaths at persistently high figures. The focus of this study was on AMTSL, a recognized gold standard strategy for prevention of postpartum hemorrhage, which is the leading cause of maternal deaths in the country.

Overall, AMTSL was a routine practice in all of the study facilities and two thirds of all the observed deliveries received Oxytocin as recommended by WHO and by the National guidelines [5] [8] [9]. The general high practice of AMTSL to prevent postpartum hemorrhage is similar to what has been observed in many developed and developing countries [11] [13]. The observation that two thirds of deliveries received Oxytocin to prevent postpartum haemorrhage within one minute of childbirth is a remarkable progress compared to the $10.4 \%$ which was reported by Cyntia et al., in Tanzania in 2005 [11], and the 7\% which was reported by Mfinanga et al. in 2008 [12]. In contrast to these two previous studies whose samples were national representative, the current results emanate from a study in an urban setting of a sub-national area where the availability of uterotinics and AMTSL training for health care workers would generally be expected to be better. Further, in contrast to the current study the study by Cyntia et al., was confined to lower delivery intensity facilities (with only one to three deliveries per day) which should have yielded better adherence to implementation of AMTSL. Thus, the current better implementation of AMTSL in Dar es Salaam could be true sign of improved quality of obstetric care services in more recent years. This connotation is further supported by the most recent study by Bartlett et al. [13] in six Sub-Saharan Africa countries with Tanzania inclusive whereby among the 706 observed deliveries in Tanzania, the correct administration of the uterotonic for AMTSL was observed in 50\%. However, the other side of these results shows that a third of delivered women did not receive Oxytocin according to the National guidelines. These women were probably at increased risk for severe postpartum hemorrhage and related morbidity and deaths [16]. More efforts are therefore needed to ensure all delivering women receive Oxytocin at the recommended time if maternal deaths and morbidity due to obstetric hemorrhage are to be minimized.

This study had its primary focus on LDIFs and HDIFs with the aim to establish the impact of delivery workload diversity on the correct implementation of 
AMTSL components and other life saving interventions. The distinction between LDIF and HDIF facilities has not been addressed by previously published studies on AMTSL in Tanzania. The results show a significant difference in the practice of AMTSL for the two levels of delivery workload with all the components of AMTSL being more likely to be performed correctly in LDIFs than HDIFs. In addition, most other important procedures during labour and childbirth were more likely to be performed in LDIFs than HDIFs. It is understood that high intensity delivery wards are likely to be congested, using overwhelmed workforce and probably exhausted supplies and medications [15]. The human resource for health in Tanzania is less than 10 per 10,000 population [15], which is below the 23 human resource for health per 10,000 population threshold for a workforce crisis [14]. Apparently improving human resource and obstetric care facility density are basic for improved life saving obstetric care delivery. It could be said that the Primary Health Services Development Program (PHSDP) in Tanzania was appropriate and focused strategy but the current study results suggest the need for more to be done in order to further reduce delivery workload to the level that will allow correct implementation of key obstetric life saving procedures. Although there was evidence of progress being made since 2005, there is still room for improvement. As new evidence becomes available and revisions to global guidelines are developed, national policies and guidelines should also be updated accordingly. Facility protocols could minimize or eliminate the practice of optional components of AMTSL especially in HDIFs in order to devote more time on the most relevant and evidence based interventions.

This study has some strengths and limitations. The direct observation and documentation of labor and delivery events gives strength to this study although the approach suffers from the risk of introducing observer bias. The approach could have led to improved performance of health care providers due to the presence of an observer, notwithstanding the efforts made to mask the primary study goal. The limit to observe deliveries occurring between $8.00 \mathrm{am}$ and $2.00 \mathrm{pm}$ was planned to uniformly evaluate one shift and a reasonable number of daily deliveries. This time limit was also designed to set comparable grounds among facilities at the working time during which medicines and other supplies have not been exhausted. This biased time limit could therefore have exaggerated the overall prevalence of Oxytocin administration due to non observance of activities during odd working hours. Fortunately, however, Oxytocin was available in adequate supplies in all the study facilities throughout the study period. Lastly, this study did not focus on the provider per client ratios which may also affect the quality of service delivery. However, the study design involved similar types of facilities on both the study groups, which reassures that the number of providers per shift was not appreciably different.

\section{Conclusion}

Facility delivery intensity has remarkable impact on performance of AMTSL and 
other life saving interventions during labor and delivery, with poorer performances being observed in HDIFs than the LDIFs. Strategies to reduce obstetric facility overload should be implemented if maternal lives are to be saved.

\section{Declarations}

\section{Ethics Approval and Consent to Participate}

This study was ethically approved by the Muhimbili University of Health and Allied Sciences Senate Research and Publication committee. Permission to conduct the study at the four health facilities was sought from the Dar es Salaam Regional Administrative Secretary (RAS) and the specific Municipal Medical officers. A written consent to participate in the study was obtained from the participants in conformity with the MUHAS senate Research and Publication committee requirements. Confidentiality and privacy were maintained during data collection, analysis and reporting.

\section{Consent to Publish}

Not applicable.

\section{Availability of Data and Materials}

The datasets generated and/or analysed during the current study are not publicly available but could be available from the corresponding author on reasonable request.

\section{Funding}

This study has been funded through a small grant provided by the Directorate of Research and Publications at Muhimbili University of Health and Allied Sciences (MUHAS).

\section{Authors' Contributions}

HK and PS contributed to the planning and design of the study. HK did field work under the supervision of PS. Both were involved in data analysis, manuscript writing, reading and approval of the final draft.

\section{Acknowledgements}

The authors of this article would like to acknowledge the contributions from members of academic staff in the Department of Obstetrics and Gynecology at MUHAS for their valuable inputs during designing and planning of the study. Acknowledgement is also made to the data collectors and healthcare providers in Dar es Salaam for their cooperation during data collection. Finally, acknowledgement is made to MUHAS for financial support.

\section{Conflicts of Interest}

The authors declare no conflicts of interest regarding the publication of this paper. 


\section{References}

[1] Royal College of Obstetricians and Gynecologists (RCOG) (2011) Prevention and Management of Post-Partum Haemorrhage: Green-Top Guidelines No. 52. RCOG, London, 1-24.

[2] Tanzania Bureau of Statistics and Macro International (2010) Tanzania Demographic and Health Survey. National Bureau of Statistics Dar es Salaam, Tanzania ICF Macro Calverton, Dar es Salaam, 1-482.

[3] Ministry of Heath, Community Development, Gender, Elderly, and Children (MOHCDGEC) (2015) Availability, Utilisation and Quality of Emergency Obstetric and Newborn Care (EmONC) Services in Tanzania Mainland.

[4] Lalonde, A (2012) Prevention and Treatment of Postpartum Hemorrhage in Low-Resource Settings. International Journal of Gynecology \& Obstetrics, 117, 108-118. https://doi.org/10.1016/j.ijgo.2012.03.001

[5] Gulmezoglu, A.M., Lumbiganon, P., Landoulsi, S., Widmer, M., Abdel-Aleem, H., Festin, M., Carroli, G., Qureshi, Z., Souza, J.P., Bergel, E., et al. (2012) Active Management of the Third Stage of Labour with and without Controlled Cord Traction: A Randomised, Controlled, Non-Inferiority Trial. The Lancet, 379, 1721-1727. https://doi.org/10.1016/S0140-6736(12)60206-2

[6] Sheldon, W.R., Durocher, J., Winikoff, B., Blum, J. and Trussell, J. (2013) How Effective Are the Components of Active Management of the Third Stage of Labor? BMC Pregnancy Childbirth, 13, 46. https://doi.org/10.1186/1471-2393-13-46

[7] Sheldon, W.R. and Winikoff, B. (2012) Controlled Cord Traction in Active Management of the Third Stage of Labour. The Lancet, 380, 1146.

https://doi.org/10.1016/S0140-6736(12)61664-X

[8] World Health Organization (2012) WHO Recommendations for the Prevention and Treatment of Postpartum Haemorrhage. Geneva.

[9] Ministry of Health and Social Welfare (MOHSW) (2008) United Republic of Tanzania Ministry of Health and Social Welfare Guidelines for Use of Uterotonics in Active Management of Third Stage of Labour.

[10] Festin, M.R., Lumbiganon, P., Tolosa, J.E., Finney, K.A., Ba-Thike, K., Chipato, T., Gaitan, H., Xu, L., Limpongsanurak, S., Mittal, S., et al. (2003) International Survey on Variations in Practice of the Management of the Third Stage of Labour. Bulletin of the World Health Organization, 81, 286-291.

[11] Cynthia Stanton, D.A., Knight, R., Ariawan, I., Gbangbade, S., Getachew, A., Portillo, J.A., Jarquin, D., Marin, F., Mfinanga, S., Vallecillo, J., Johnsona, H. and Sintasatha, D. (2009) Use of Active Management of the Third Stage of Labour in Seven Developing Countries. Bulletin of the World Health Organization, 87, 207-215. https://doi.org/10.2471/BLT.08.052597

[12] Mfinanga, G.S., Kimaro, G.D., Ngadaya, E., Massawe, S., Mtandu, R., Shayo, E.H., Kahwa, A., Achola, O., Mutungi, A., Knight, R., et al. (2009) Health Facility-Based Active Management of the Third Stage of Labor: Findings from a National Survey in Tanzania. Health Research Policy and Systems, 7, 6. https://doi.org/10.1186/1478-4505-7-6

[13] Bartlett, L., Cantor, D., Lynam, P., Kaur, G., Rawlins, B., Ricca, J., Tripathi, V. and Rosen, H.E. (2015) Facility-Based Active Management of the Third Stage of Labour: Assessment of Quality in Six Countries in Sub-Saharan Africa. Bulletin of the World Health Organization, 93, 759-767. https://doi.org/10.2471/BLT.14.142604

[14] World Health Organisation (2006) The World Health Report 2006: Working To- 
gether for Health.

[15] Ministry of Health and Social Welfare (MOHSW) (2014) Human Resource for Health and Social Welfare Strategic Plan 2014-2019. Ministry of Health and Social Welfare, Dar es Salaam.

[16] Soltani, H., Hutchon, D.R. and Poulose, T.A. (2010) Timing of Prophylactic Uterotonics for the Third Stage of Labour after Vaginal Birth. Cochrane Database of Systematic Reviews, No. 8, CD006173.

https://doi.org/10.1002/14651858.CD006173.pub2 


\section{Abbreviations}

AMTSL: Active Management of Third Stage of Labour; FIGO: International Federation of Gynecology and Obstetrics; HDIF: High Delivery Intensity Facility; ICM: International Confederation of Midwives; LDIF: Low Delivery Intensity Facility; MUHAS: Muhimbili University of Health and Allied Sciences; PHSDP: Primary Health Services Development Program; PPH: Postpartum Hemorrhage; RAS: Regional Administrative Secretary.

\section{Appendix}

\section{Checklist (English Version)}

1) Name of the facility

2) Registration number of the patient

3) Gravidity

4) Parity

5) Gestational age at time of delivery (in weeks)

6) Antenatal history:-

a) Number of antenatal visits made

b) Last recorded hemoglobin level

c) Last recorded blood pressure [Systole....../Diastole ( $\mathrm{mmHg})$

d) PMTCT status: 1/2/0

e) Number of Anti malaria doses received:

f) Hematinics doses received

7) Socio demographic factors

a) Maternal age at time of delivery (completed years)

b) Place of residence.

c) Marital status:-

Single

Married

Divorced

Separated

d) Highest Level of Education:-

No formal education

Standard seven

Form four

Form six

College/University

8) Intrapartum History: -

a) Initiation of labor

Spontaneous $\rightarrow$ Skip ii \& iii below

Induced

b) If labor induced, how

Misoprostol

Dinoprostone 


\section{Oxytocin}

Mechanically, balloon catheter

Other.

c) Attempts made at labor induction

One

Two

More than two

d) Was labor augmented?

Yes

No $\rightarrow$ skip v below

e) Dose of oxytocin used for augmentation of labor

$5 \mathrm{IU}$

$2.5 \mathrm{IU}$

$1.25 \mathrm{IU}$

f) Was a partograph filled?

Yes

$\square$ No

g) Was the patient catheterized?

$\square$ Yes

No

h) Did the patient receive any i.v fluids during the course of labor

$\square$ Yes

$\square$ No

i) If i.v fluids given, how many liters in total

j) Type of i.v fluids given

$\square$ Ringers lactate

$\square$ Normal saline

Dextrose normal saline

$5 \%$ dextrose solution

$10 \%$ dextrose solution

k) Was episiotomy done

Yes

No

1) Was operative vaginal delivery used to assist delivery?

Yes

No

m) If yes, type of operative vaginal delivery used

Vacuum

Forceps

9) Time of delivery

10) Observed time of delivery of the baby (last baby if twins)

11)Was any oxytocic given after delivery (through 3 hours after delivery?)

Yes

No 
12)If given, what type?

Oxytocin

$\square$ Ergomatrine

Misoprostol

Other, mention

13)If given, how many counted minutes from time of delivery to administration of the Oxytocic medicine? [record stop-watch timing from the time the last babies body part was delivered/not the placenta]? [N/A if not recorded].

14)Was controlled cord traction done?

Done

Not done

15)If done, how properly was it done?

Perfect

Mainly correct

Somehow correct

Totally incorrect

16) Was the placenta and its membranes delivered complete?

$\square$ Yes

$\square$ No

17) Was uterine massage attempted?

$\square$ Yes

No

18)If uterine massage was done, how properly was it done?

Perfect

Mainly correct

Somehow correct

Totally correct

19)What was the duration of the third stage of labor [From records of complete baby delivery to complete delivery of placenta]? (Minutes)

20)How much was the observed blood loss post-delivery?

Normal loss

Slightly abnormally large amount but woman looks normal without intravenous fluids

$\square$ Excessive blood loss with sick woman needing i.v fluids or blood for rescusitation

21) Any HCW in attendance during spontaneous delivery?

$\square$ Yes

No

22)If HCW was present, what was the cadre?

Midwife

Registered nurse

Health attendant 
$\square$ Medical doctor

23) Any complications observed within 3 hours of delivery [tick all that were observed]?

Uterine atony

$\square$ Postpartum hemorrhage

$\square$ Hypotension

$\square$ Hypovolemic shock

$\square$ Perineal hematoma

$\square$ Fever

$\square$ Pre-eclampsia/eclampsia

$\square$ Other.. 